\title{
Sentinel lymph node biopsy in upper tract urothelial cancers: an experience with intraoperative radiotracer injection
}

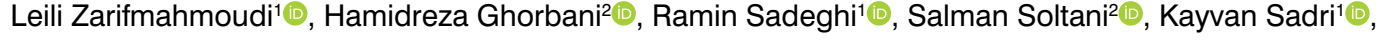 \\ Mahmoud Tavakkoli²), Maliheh Keshvari2 ${ }^{2}$ \\ ${ }^{1}$ Nuclear Medicine Research Center, Mashhad University of Medical Sciences, Mashhad, Iran \\ ${ }^{2}$ Kidney Transplantation Complications Research Center, Mashhad University of Medical Sciences, Mashhad, Iran
}

[Received 7 V 2020; Accepted 28 VI 2021]

\begin{abstract}
Background: The feasibility of the sentinel node mapping in upper tract urothelial cancers (UTUC) was evaluated, using a radiotracer as the mapping material.
\end{abstract}

Material and methods: To identify the sentinel lymph nodes, $37 \mathrm{MBq}$ of [99mTc] phytate was injected in five patients with the renal pelvis or ureter cancer, who were candidates for ureterectomy and lymphadenectomy. The radiotracer was injected in a peritumoral fashion following the surgical exposure of the tumour. The sentinel lymph nodes were detected using a handheld gamma probe.

Results: By intraoperatively injecting the radiotracer immediately after surgical exposure of the tumour, at least one sentinel lymph node could be detected in each patient, and the detection rate was $100 \%$. The location of sentinel nodes was in the paracaval, renal hill, retro-aortic, para-aortic, common iliac, and external iliac areas, which was dependent on the tumour location. No false-negative case was identified.

Conclusions: Sentinel node mapping is feasible in UTUC. Injection technique (intra-vesical approach vs peri-tumoral injection after exposure of the tumour) and location of the tumour (proximal vs distal) may affect the technique's feasibility.

KEY words: lymphoscintigraphy; nuclear medicine; ureter; renal pelvis; sentinel lymph node biopsy; lymphadenectomy

Nucl Med Rev 2021; 24, 2: 41-45

\section{Introduction}

Urothelial carcinomas can be originated from lower (bladder and urethra) or upper (pyelocaliceal cavities and ureter) parts of the urinary tract [1]. Bladder carcinomas (lower tract urothelial carcinomas) account for 90-95\% of the urinary tract malignancies; however, the upper urinary tract (UUT) transitional cell carcinoma (TCC) is an uncommon type of malignancies and accounts for $5-10 \%$ of urinary tract cancers [2,3]. Tumours of the ureter are particularly rare compared to bladder cancer or renal pelvis tumours, despite sharing common histopathology and similar risk factors [4].

Correspondence to: Hamidreza Ghorbani

Kidney Transplantation Complications Research Center, Mashhad University of Medical Sciences, Mashhad, Iran

e-mail: Ghorbanihr@mums.ac.ir
The most common treatment option for high-grade tumours originate in the upper ureter and renal pelvis, is nephroureterectomy followed by regional lymph node dissection (LND); however, due to the low incidence of the upper tract urinary carcinoma (UTUC), the indication and extent of LND is not standardized [5]. The risk of lymph node involvement increases with the $T$ stage of the disease, and up to $60 \%$ of UTUCs demonstrate local invasion at diagnosis [6]. LND is recommended to be performed based on the laterality and tumour location following nephroureterectomy; however, due to variable lymphatic drainage and lack of consensus on anatomical boundaries, the potential benefit of routine LND on survival or disease recurrence remains controversial $[7,8]$.

Sentinel lymph node mapping procedure is used for detecting the first lymph node in the path of lymphatic drainage of cancers. This technique reveals the pathological status of the regional lymph nodes for the better staging of the disease, determining the treatment strategy, and preventing the unnecessary LND. Sentinel lymph node biopsy is routinely performed for breast cancer, 
malignant melanoma, gynaecological, and penile tumours; however, for other urologic genitourinary carcinomas, it is still under investigation [9-14].

The present study evaluated the feasibility of sentinel node mapping in UTUC using a radiotracer as the mapping material.

\section{Material and methods}

This study was approved by the ethics committee of Mashhad University of Medical Sciences under the number 940295. Between 2016 and 2019, all the admitted UTUC patients at the urology department of Imam Reza Hospital, Mashhad, Iran, were included. All the patients gave their informed consent before inclusion in the study. Patients with negative lymph node involvement before the surgery, based on clinical and radiological evaluations (cNO, cM0) were included. The exclusion criteria were positive lymph nodes metastasis and a history of chemotherapy or radiotherapy.

\section{Radiotracer injection}

Following anaesthesia, $37 \mathrm{MBq}$ of [99m Tc] phytate in two divided doses $(0.5 \mathrm{~mL}$ each) were used as the radiotracer. In the first four patients with TCC of the distal part of the ureter, the radiotracer was injected sub-mucosally in the peritumoral area of the tumour through ureteroscopy. These patients were excluded from the study due to sentinel lymph node detection failure. The injection technique was changed in five other patients: the radiotracer was injected into two peritumoral sites following the tumour's exposure (Tab. 1, Fig. 1).

\section{Sentinel node biopsy}

The mean time between the tracer injection and performing intraoperative sentinel lymph node mapping was one hour. Following the excision of the ureter and kidney and before starting lymphadenectomy, a hand-held gamma probe (SURGIGUIDE, Partonegar Persia) was used to measure the radioactivity and perform sentinel node biopsy. The sentinel lymph nodes were defined as any lymph node with an in-vivo count of at least ten times higher than the background (thigh). These nodes were dissected and counted again ex-vivo. The dissected lymph nodes were put on

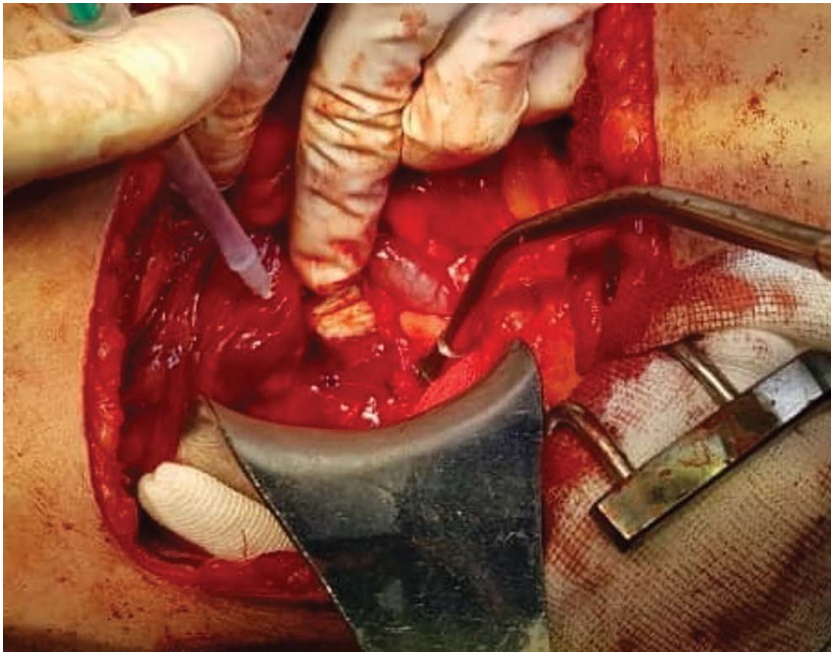

Figure 1. Injection of the radiotracer in a patient with mid-urethral tumour, after the surgergical exposure of the tumour

the tip of the gamma probe while the probe was pointed to the ceiling. All the lymph nodes with ex-vivo count five times higher than the background (thigh) were eventually determined as the sentinel lymph node.

After harvesting the detected sentinel lymph nodes, the surgical area was searched again to confirm the complete sentinel lymph node removal.

Regional lymphadenectomy was performed based on the surgeon's decision to assess the false-negative rate. For the left-sided renal pelvis, upper ureteral, and mid-urethral tumours, the lymphadenectomy dissection area covered para-aortic, pre-aortic, and inter-aortocaval nodes from the renal hilum to the aortic bifurcation. For the right-sided renal pelvis and upper ureteral tumours, mid-urethral tumours lymph nodes of the paracaval, precaval, and interaortocaval areas from the renal hilum to the aortic bifurcation are considered for lymphadenectomy. Common iliac, external iliac, obturator, and hypogastric lymph nodes of each side of the pelvic are also dissected for mid to distal ureteral tumours.

\section{Table 1. Detailed data of the included patients}

\begin{tabular}{|c|c|c|c|c|c|c|c|c|c|c|c|}
\hline Patients & Gender & age & Tumor location & kidney & $\begin{array}{l}\text { Tumor } \\
\text { stage }\end{array}$ & $\begin{array}{l}\text { Tumor larger } \\
\text { diameter } \\
\text { (cm) }\end{array}$ & $\begin{array}{l}\text { Detection } \\
\text { of the SN }\end{array}$ & $\begin{array}{l}\text { SN loca- } \\
\text { tion }\end{array}$ & $\begin{array}{l}\text { Number } \\
\text { of SN }\end{array}$ & $\begin{array}{l}\text { Involvement } \\
\text { of SN }\end{array}$ & $\begin{array}{l}\text { Involvement of } \\
\text { lymph nodes }\end{array}$ \\
\hline 1 & M & 53 & Middle & right & T3 & 2.5 & Yes & Paracaval & 3 & Yes $1 / 3$ & Yes (paracaval) \\
\hline 2 & M & 74 & Proximal & Left & $\mathrm{T} 1$ & 2.7 & Yes & Renal hill & 2 & No & No \\
\hline 3 & $M$ & 40 & $\begin{array}{l}\text { Renal pelvis } \\
\text { (proximal) }\end{array}$ & Right & T3 & $4 \times 2$ & Yes & Renal hill & 2 & No & No \\
\hline 4 & M & 80 & Distal & Right & T2 & 4.5 & Yes & $\begin{array}{l}\text { Common } \\
\text { iliac } \\
\text { external } \\
\text { iliac }\end{array}$ & 2 & No & No \\
\hline 5 & $\mathrm{~F}$ & 48 & $\begin{array}{l}\text { Renal pelvis } \\
\text { (proximal) }\end{array}$ & left & T2 & 4 & Yes & $\begin{array}{l}\text { Retroaort } \\
\text { and } \\
\text { Paraaort }\end{array}$ & 2 & No & No \\
\hline
\end{tabular}




\section{Histopathological investigation}

The pathologists examined the excised lymph nodes and specimen; all dissected tissues were formalin-fixed, paraffin-embedded, and serially sectioned for haematoxylin-eosin staining. The histopathological status of the sentinel nodes was compared with the other dissected lymph nodes.

\section{Evaluated indices (detection rate and false-negative rate)}

The primary endpoints of the study were sentinel node detection rate and false-negative rate (FNR).

The detection rate was measured as the ratio of all cases with at least one detected sentinel node to all included patients. The false-negative rate was measured as the ratio of patients with involved non-sentinel lymph nodes despite pathologically free sentinel lymph nodes to all patients with involved nodes and at least one harvested sentinel node $[15,16]$.

\section{Results}

Five patients with renal pelvis or ureter cancer were included; four patients were male, and one was female with a mean age of 59 .

The tumour was localized in proximal ureter in two patients, distal ureter in one patient, and middle ureter in one patient.

Demographic data and information regarding the sentinel lymph node detection are summarized in Table 1.

\section{Detection rate}

In all patients with the peritumorally injection of the radiotracer following the surgical exposure of the tumour, at least one sentinel node was detected. The detection rate was 100\% in these five patients, and eleven (median $=2$ ) sentinel lymph nodes were dissected in total (Fig. 1).

All the patients with no detected sentinel node were injected through the ureteroscopy at two sites around the visible tumour; so, the detection rate was zero in UTUC patients with ureteroscopic injections; these patients were excluded from the study.

The detected sentinel nodes were in the paracaval lymph nodes (patient 1), renal hilum lymph nodes (patients 2 and 3), retro aortic and para-aortic lymph nodes (patient 5), and common iliac and external iliac lymph nodes (patient 4).

\section{False-negative rate}

One patient (number 1) had pathological involvement of the dissected sentinel lymph node. The detected sentinel nodes were in paracaval area (three sentinel nodes), and one showed tumoral involvement. Based on the pathology report, the non-sentinel paracaval lymph nodes dissected in this patient were also pathologically involved.

No lymph node involvement was noted in the remaining four patients (no false-negative result).

\section{Discussion}

Upper urinary tract TCCs are among the rare types of genitourinary tumours which develop in the renal pelvis and ureter and might be associated with bladder cancer. To the authors' knowledge, they evaluated the feasibility of the sentinel node mapping approach for the first time in patients with UTUC including, patients with TCCs in the proximal, middle, and distal parts of the ureter and also in the renal pelvis. They used Tc-99m phytate as the mapping material, and no blue dye was used due to the reported adverse reaction to the blue dye injection $[17,18]$.

The detection rate in this study was highly dependent on the injection method of the radiotracer. In patients with the ureteroscopic injection of the tracer, no sentinel node could be identified intra-operatively. So, these patients were excluded from the study and changed the injection technique. In the remaining patients (five patients) with radiotracer injection following the tumour exposure during the surgery, at least one sentinel node could be identified. These results are most likely due to the technical difficulty of injection through a ureteroscope. The movement of the radiotracer in the lymphatic system is reasonably fast, which ensures a successful mapping using an intra-operative injection of the tracer [19-21].

Although LND is performed for better staging of the tumour, due to the low prevalence of UTUC, the curative role of LND continues to be debated, and LND is not currently performed in all patients worldwide. In patients with the T1 stage of the disease, there might be a low probability of the LN metastases, and LND does not seem to improve the staging. All the current evidence on UTUC is based on retrospective studies with a limited study population. To precisely determine the indication of LND and its optimal template, which varies according to the location of the disease, more extensive studies should be performed [22-27]. Although the LND template has been studied previously, there is no standardized template for $L N D$, and it is dependent on the site of the affected ureter $[1,28]$.

Based on the present results, sentinel lymph nodes as the first lymph nodes which receive the metastatic cells were located in the renal hilum, retro-aortic, and para-aortic lymph nodes of the patients with TCCs in the proximal part of the left ureter or the left renal pelvis. However, in two patients with TCCs in the middle part of the right ureter and the right renal pelvis, the detected sentinel lymph nodes were in paracaval and right renal hilum lymph nodes. The sentinel lymph nodes in one of the study patients with TCC in the distal part of the right ureter was in the common iliac and external iliac lymph nodes. The present results were in accordance with the previous studies in this regard. Kondo et al. [22] proposed that the paracaval, retrocaval, and interaortocaval nodes have a higher risk of metastases in patients with the right renal pelvis and upper two-thirds of the ureter tumours and should be included in the LND template. On the other hand, the renal hilum and para-aortic nodes in patients with left renal pelvis tumours should be considered in the LND template.

We included only patients with no lymph node involvement based on preoperative imaging; however, these modalities have shown limited accuracy for preoperative LN staging or identifying patients with LN invasion [29, 30]. LND in patients with UTUC is highly surgeon-dependent due to inaccurate prediction tools, and sentinel lymph node mapping might be of value to find the metastatic status of the regional lymph nodes. This study showed that sentinel node could be detected in all the UTUC patients with the intraoperative injection of the radiotracer mapping, and sentinel lymph node mapping might be a feasible method in patients with tumours in different parts of the ureter or renal pelvis. However, more extensive studies (preferably multicentral) are needed before any definitive conclusion. 
The major limitation of the present study was the low sample size due to the low incidence of UTUC. However, to the authors' knowledge, this study is the first study on intraoperative sentinel lymph node mapping in UTUC tumours, which obtained promising results.

\section{Conclusions}

Sentinel node mapping is feasible in UTUC. Injection technique (intra-vesical approach vs peri-tumoral injection after exposure of the tumour) and location of the tumour (proximal vs distal) might affect the technique's feasibility.

\section{References}

1. Rouprêt M, Babjuk M, Compérat E, et al. European Association of Urology guidelines on upper urinary tract urothelial carcinoma: 2017 update. Eur Urol. 2018; 73(1): 111-122, doi: 10.1016/j.eururo.2017.07.036, indexed in Pubmed: 28867446.

2. Babjuk M, Böhle A, Burger M, et al. EAU guidelines on non-muscle-invasive urothelial carcinoma of the bladder: update 2016. Eur Urol. 2017; 71(3): 447-461, doi: 10.1016/j.eururo.2016.05.041, indexed in Pubmed: 27324428.

3. Siegel RL, Miller KD, Jemal A. Cancer Statistics, 2017. CA Cancer J Clin. 2017; 67(1): 7-30, doi: 10.3322/caac.21387, indexed in Pubmed: 28055103.

4. Greenlee RT, Murray T, Bolden S, et al. Cancer statistics, 2000. CA Cancer J Clin. 2000; 50(1): 7-33, doi: 10.3322/canjclin.50.1.7, indexed in Pubmed: 10735013

5. Spiess PE, Agarwal N, Bangs R, et al. Bladder cancer, version 5.2017, NCCN clinical practice guidelines in oncology. J Natl Compr Canc Netw. 2017; 15(10): 1240-1267, doi: 10.6004/jnccn.2017.0156, indexed in Pubmed: 28982750.

6. Leow JJ, Liu Z, Tan TW, et al. Optimal management of upper tract urothelial carcinoma: current perspectives. Onco Targets Ther. 2020; 13: 1-15, doi: 10.2147/OTT.S225301, indexed in Pubmed: 32021250.

7. Stein JP, Lieskovsky G, Cote R, et al. Radical cystectomy in the treatment of invasive bladder cancer: long-term results in 1,054 patients. J Clin Oncol. 2001; 19(3): 666-675, doi: 10.1200/JCO.2001.19.3.666, indexed in Pubmed: 11157016.

8. Zigeuner R, Pummer K. Urothelial carcinoma of the upper urinary tract: surgical approach and prognostic factors. Eur Urol. 2008; 53(4): 720-731, doi: 10.1016/j.eururo.2008.01.006, indexed in Pubmed: 18207315

9. Zarifmahmoudi L, Ghorbani H, Sadri K, et al. Sentinel node biopsy in urothelial carcinoma of the bladder: systematic review and meta-analysis. Urol Int. 2019; 103(4): 373-382, doi: 10.1159/000497310, indexed in Pubmed: 30836375.

10. Hassanzade M, Attaran M, Treglia G, et al. Lymphatic mapping and sentinel node biopsy in squamous cell carcinoma of the vulva: systematic review and meta-analysis of the literature. Gynecol Oncol. 2013; 130(1): 237-245, doi: 10.1016/j.ygyno.2013.04.023, indexed in Pubmed: 23612317.

11. Sadeghi R, Gholami H, Zakavi SR, et al. Accuracy of sentinel lymph node biopsy for inguinal lymph node staging of penile squamous cell carcinoma: systematic review and meta-analysis of the literature. J Urol. 2012; 187(1): 25-31, doi: 10.1016/j.juro.2011.09.058, indexed in Pubmed: 22088350.

12. Kadkhodayan S, Hasanzadeh M, Treglia G, et al. Sentinel node biopsy for lymph nodal staging of uterine cervix cancer: a systematic review and meta-analysis of the pertinent literature. Eur J Surg Oncol. 2015; 41(1): 1-20, doi: 10.1016/j.ejso.2014.09.010, indexed in Pubmed: 25454828.

13. Sadeghi R, Tabasi KT, Bazaz SMM, et al. Sentinel node mapping in the prostate cancer. Meta-analysis. Nuklearmedizin. 2011; 50(3): 107-115, doi: 10.3413/nukmed-0339-10-07, indexed in Pubmed: 21264441.
14. Ansari M, Rad MA, Hassanzadeh M, et al. Sentinel node biopsy in endometrial cancer: systematic review and meta-analysis of the literature. Eur J Gynaecol Oncol. 2013; 34(5): 387-401, indexed in Pubmed: 24475571.

15. Sadeghi R. Sentinel node mapping diagnostic studies warrant a unique reporting criteria: comment on Xiong et al. systematic review. Eur J Surg Oncol. 2014; 40(8): 1025-1026, doi: 10.1016/j.ejso.2014.03.031, indexed in Pubmed: 24857379.

16. Zarifmahmoudi $L$, Sadeghi $R$. Re: radio-guided lymph node mapping in bladder cancer using SPECT/CT and intraoperative -Probe methods. Clin Nucl Med. 2017; 42(4): 327, doi: 10.1097/RLU.0000000000001485, indexed in Pubmed: 27997419.

17. Ramin S, Azar FP, Malihe H. Methylene blue as the safest blue dye for sentinel node mapping: emphasis on anaphylaxis reaction. Acta Oncol. 2011; 50(5): 729-731, doi: 10.3109/0284186X.2011.562918, indexed in Pubmed: 21413854.

18. Jangjoo A, Forghani MN, Mehrabibahar M, et al. Anaphylaxis reaction of a breast cancer patient to methylene blue during breast surgery with sentinel node mapping. Acta Oncol. 2010; 49(6): 877-878, doi: 10.3109/02841861003769964, indexed in Pubmed: 20429734.

19. Sadeghi R, Forghani MN, Memar B, et al. How long the lymphoscintigraphy imaging should be continued for sentinel lymph node mapping? Ann Nucl Med. 2009; 23(6): 507-510, doi: 10.1007/s12149-009-0284-y, indexed in Pubmed: 19588215.

20. Aliakbarian M, Memar B, Jangjoo A, et al. Factors influencing the time of sentinel node visualization in breast cancer patients using intradermal injection of the radiotracer. Am J Surg. 2011; 202(2): 199-202, doi: 10.1016/j. amjsurg.2010.06.035, indexed in Pubmed: 21810501.

21. Jangjoo A, Forghani MN, Mehrabibahar M, et al. Comparison of early and delayed lymphoscintigraphy images of early breast cancer patients undergoing sentinel node mapping. Nucl Med Commun. 2010; 31(6): 521-525, indexed in Pubmed: 20429094.

22. Kondo $\mathrm{T}$, Nakazawa $\mathrm{H}$, Ito $\mathrm{F}$, et al. Impact of the extent of regional lymphadenectomy on the survival of patients with urothelial carcinoma of the upper urinary tract. J Urol. 2007; 178(4 Pt 1): 1212-1217, doi: 10.1016/j. juro.2007.05.158, indexed in Pubmed: 17698147.

23. Brausi MA, Gavioli M, De Luca G, et al. Retroperitoneal lymph node dissection (RPLD) in conjunction with nephroureterectomy in the treatment of infiltrative transitional cell carcinoma (TCC) of the upper urinary tract: impact on survival. Eur Urol. 2007; 52(5): 1414-1418, doi: 10.1016/j.eururo.2007.04.070, indexed in Pubmed: 17507148.

24. Roscigno M, Cozzarini C, Bertini R, et al. Prognostic value of lymph node dissection in patients with muscle-invasive transitional cell carcinoma of the upper urinary tract. Eur Urol. 2008; 53(4): 794-802, doi: 10.1016/j. eururo.2008.01.008, indexed in Pubmed: 18207313.

25. Roscigno M, Shariat SF, Freschi M, et al. Assessment of the minimum number of lymph nodes needed to detect lymph node invasion at radical nephroureterectomy in patients with upper tract urothelial cancer. Urology. 2009; 74(5): 1070-1074, doi: 10.1016/j.urology.2009.04.084, indexed in Pubmed: 19883824.

26. Roscigno M, Shariat SF, Margulis V, et al. The extent of lymphadenectomy seems to be associated with better survival in patients with nonmetastatic upper-tract urothelial carcinoma: how many lymph nodes should be removed? Eur Urol. 2009; 56(3): 512-518, doi: 10.1016/j.eururo.2009.06.004, indexed in Pubmed: 19559518.

27. Miyake H, Hara I, Gohji K, et al. The significance of lymphadenectomy in transitional cell carcinoma of the upper urinary tract. Br J Urol. 1998; 82(4): 494-498, doi: 10.1046/j.1464-410x.1998.00800.x, indexed in Pubmed: 9806176.

28. Roscigno M, Brausi M, Heidenreich A, et al. Lymphadenectomy at the time of nephroureterectomy for upper tract urothelial cancer. European Urology. 2011; 60(4): 776-783, doi: 10.1016/j.eururo.2011.07.009. 
29. Secin FP, Koppie TM, Salamanca JI, et al. Evaluation of regional lymph node dissection in patients with upper urinary tract urothelial cancer. Int $\mathrm{J}$ Urol. 2007; 14(1): 26-32, doi: 10.1111/j.1442-2042.2006.01664.x, indexed in Pubmed: 17199856
30. Lughezzani $\mathrm{G}$, Jeldres $\mathrm{C}$, Isbarn $\mathrm{H}$, et al. A critical appraisal of the value of lymph node dissection at nephroureterectomy for upper tract urothelial carcinoma. Urology. 2010; 75(1): 118-124, doi: 10.1016/j.urology.2009.07.1296, indexed in Pubmed: 19864000 